\title{
Overexpression of Mortalin in hepatocellular carcinoma and its relationship with angiogenesis and epithelial to mesenchymal transition
}

\author{
JING CHEN $^{1 *}$, WEN-BIN LIU ${ }^{1 *}$, WEI-DONG JIA ${ }^{1}$, GE-LIANG XU ${ }^{1}$, JIN-LIANG MA $^{1}$, \\ MEI HUANG ${ }^{2}$, YAN-RU DENG ${ }^{3}$ and JIAN-SHENG LI ${ }^{1}$ \\ ${ }^{1}$ Department of Hepatic Surgery, ${ }^{2}$ Anhui Province Key Laboratory of Hepatopancreatobiliary Surgery, \\ ${ }^{3}$ Laboratory of Emergency Medicine, Anhui Provincial Hospital Affiliated to \\ Anhui Medical University, Hefei, Anhui 230001, P.R. China
}

Received August 31, 2013; Accepted October 10, 2013

DOI: $10.3892 /$ ijo.2013.2161

\begin{abstract}
Mortalin is highly expressed in a variety of human tumors and associated with tumor metastasis. However, the relationship among the overexpression of Mortalin, epithelial to mesenchymal transition (EMT) and neovascularization is largely unknown. The aim of the present study was to investigate the expression of Mortalin in human HCC cell lines, clinical HCC specimens and its association with angiogenesis and EMT. The results of our study showed that the expression levels of Mortalin in cell lines with higher metastatic potential were significantly higher compared to those with lower metastatic potential. Compared with paracarcinomatous tissues and normal liver tissues, the expression of Mortalin was significantly increased in HCC tumor tissues. The expression of Mortalin was correlated with invasion and metastasis, Edmondson grade and TNM stage. A significant positive correlation was found between the expression of Mortalin and Vimentin, and tumors with high expression of Mortalin had a tendency to higher MVD compared to those with low expression of Mortalin. Using shRNA-mediated Mortalin knockdown, we found that decreased expression of Mortalin was accompanied by a reduction of Vimentin expression. Our findings demonstrated that the overexpression of Mortalin is correlated with the metastatic phenotype of HCC cells and can promote EMT, but cannot induce angiogenesis in HCC. The decreased expression of Mortalin is accompanied by an inhibition of EMT in the HCC cell lines.
\end{abstract}

Correspondence to: Dr Jian-Sheng Li, Department of Hepatic Surgery, Anhui Provincial Hospital Affiliated to Anhui Medical University, 17 Lujiang Road, Hefei, Anhui 230001, P.R. China

E-mail: 1ijiansheng1953@gmail.com

${ }^{*}$ Contributed equally

Key words: Mortalin, hepatocellular carcinoma, epithelial-mesenchymal transition, Vimentin, angiogenesis

\section{Introduction}

Hepatocellular carcinoma (HCC) is currently the sixth most common malignancy and the third leading cause of cancerrelated death worldwide, with approximately 695,900 deaths in 2008 (1,2). Although hepatectomy, liver transplantation, and ablation are the available treatment options for patients with HCC, high postoperative metastasis or recurrence rate remain the major obstacles that influence long-term survival, while most recurrences are due to invasion-related spreading. Therefore, there is an urgent need for a better understanding of the molecular mechanisms that contribute to HCC metastasis and for new potential therapeutic strategy.

Different molecular events and phenotypic changes are involved in cancer cell disintegration and migration into distant organs or tissues. The epithelial-to-mesenchymal transition (EMT), which is characterized by the loss or downregulation of epithelial markers (E-cadherin) and upregulation of mesenchymal markers (Vimentin), is a crucial step in tumor invasion and metastasis $(3,4)$. Furthermore, HCC is a hypervascular tumor and neovascularization is a common phenomenon. The development of neovasculature plays an important role in the growth and metastasis of HCC (5).

Mortalin, which is also known as heat shock protein 75 (HSP75), is low or undetectable in the normal tissues, while highly expressed in several epithelial carcinomas $(6,7)$. Overexpression of Mortalin interacts with the wild-type tumor suppressor protein p53 and modulates the Ras-Raf-MAPK pathway and then increase the malignancy of tumor cells (8-10). Therefore Mortalin appears to play a crucial role in tumorigenesis and metastasis. Recently, it was reported that Mortalin overexpression in HCC was associated with venous infiltration and advanced tumor stages and thus Mortalin was implicated as a tumor marker for predicting early recurrence (11). Nevertheless, there are no previous studies on the role of Mortalin in HCC, EMT and angiogenesis.

In the present study, we observed that the high expression of Mortalin is an important regulator inducing EMT, but it does not promote angiogenesis in HCC. Furthermore, we demonstrated that the decreased expression of Mortalin is 
accompanied by an inhibition of EMT in the HCC cell line MHCC97H.

\section{Materials and methods}

Cell culture. Five human hepatoma-derived cell lines (Hep3B, MHCC97H, HepG2, MHCC97L and HCCLM3) with various metastatic potentials and a normal liver cell line L02 were purchased from Liver Cancer Institute of Zhongshan Hospital, Shanghai, China. MHCC97-L, MHCC97-H and HCCLM3 belonged to the same genetic background and had a stepwise increasing metastatic potential $(12,13)$. The cells were maintained in Dulbecco's modified Eagle's medium (DMEM) (Gibco, NY, USA) with 10\% (vol/vol) fetal bovine serum (FBS) (Hyclone, Logan, UT, USA), $100 \mathrm{U} / \mathrm{ml}$ penicillin and $100 \mathrm{U} / \mathrm{mll}$ streptomycin at $37^{\circ} \mathrm{C}$ in a humidified incubator supplemented with $5 \% \mathrm{CO}_{2}$.

Patients and tissue samples. Tumor and paracarcinomatous tissues for immunohistochemistry were obtained from 96 patients undergoing curative hepatectomy for $\mathrm{HCC}$ at Department of Hepatic Surgery, Anhui Provincial Hospital Affiliated to Anhui Medical University from June, 2007 to June, 2010. Frozen HCC tumor and corresponding paracarcinomatous tissues were obtained from 13 patients for $\mathrm{qPCR}$ and western blotting. Ten normal liver samples were acquired from the patients who had received an operation due to hepatic trauma as the controls. No patient had received adjuvant therapies before surgery. These patients included 76 males and 24 females with a mean age of 56.4 years (range, 21-80 years). Tumor size was measured as the largest dimension of the tumor by gross examination; the pathological tumor stage was defined according to the sixth edition of the tumor-nodemetastasis (TNM) classification of the International Union Against Cancer; tumor differentiation was defined according to the Edmondson grading system (14). All tissue diagnoses were confirmed by three experienced pathologist who were unaware of patients' clinical and laboratory data. Clinicopathological parameters of the HCC patients are described in Table I. The study protocol conformed to the ethical guidelines of the declaration of Helsinki. Ethics approval for the study of human subjects was obtained from the research ethics committee of Anhui Provincial Hospital Affiliated to Anhui Medical University and written informed consent was obtained from all the patients.

RNA extraction, reverse transcription and real-time quantitative PCR $(q P C R)$. Total RNA was extracted from six cell lines, normal liver tissues, snap-frozen HCC tumor and corresponding paracarcinomatous tissues using the TRIzol reagent (Invitrogen, Carlsbad, CA, USA). The quality of mRNA was evaluated by the OD260/OD280 ratio and samples were used only when the ratio was between 1.8 and 2.0. cDNA was synthesized according to the instructions of the Revert Aid First Strand cDNA synthesis kit (Invitrogen). The sequences of Mortalin-specific primers were designed as follows: forward, 5'-GAGAGACAGGGGTTGATTTGAC-3'; reverse, 5'-GCA CAGATGAGGAGAGTTCACA-3'. In addition, the sequences of Vimentin-specific primers were designed as follows: forward, 5'-CCGACACTCCTACAAGATTTAGA-3'; reverse,
5'-CAAAGATTTATTGAAGCAGAACC-3'. For standardization of RNA quality control, the expression of glyceraldehyde3-phosphate dehydrogenase (GAPDH) in each sample was quantified by using the primer set 5'-AAGGTCATCCCTG AGCTGAAC-3' (forward) and 5'-ACGCCTGCTTCACCACC TTCT-3' (reverse). Real-time quantitative PCR was performed at $95^{\circ} \mathrm{C}$ for $5 \mathrm{sec}$ and $60^{\circ} \mathrm{C}$ for $30 \mathrm{sec}$ for 40 cycles using SYBR Green PCR Master Mix (Applied Biosystems) and Applied Biosystems 7500 Real-Time PCR System. Relative gene expression level was calculated by using the $2^{-\Delta \Delta C t}$ method. After 40 cycles of PCR, a melting curve analysis was performed to monitor PCR product purity and then the amplicons were analyzed on a $1.0 \%$ agarose gel containing ethidium bromide.

Western blot analysis. Six cultured cell lines in an exponentially growing phase were washed thoroughly twice with PBS and then proteins of cell lines, liver tissues, snap-frozen HCC and corresponding paracarcinomatous tissues were extracted using Total Protein Extraction kit (KeyGen, China). After centrifugation, protein concentrations of lysate were quantified using BCA method. Then, equal amounts of protein samples were loaded onto $10 \%$ sodium dodecyl sulfate-polyacrylamide electrophoresis (SDS-PAGE) gel and electrophoretically transferred onto polyvinylidene difluoride (PVDF) membranes (0.45 $\mu \mathrm{m}$, Millipore, MA, USA). Membranes were blocked with 5\% non-fat milk in Tris-buffered saline containing $0.1 \%$ Tween-20 (TBST) for $1 \mathrm{~h}$ and then incubated with primary antibody of rabbit anti-Mortalin antibody (ab53098) (Abcam, Cambridge, MA, USA), rabbit anti-Vimentin antibody (ab92547) (Abcam) and rabbit anti- $\beta$-actin antibody (ab133626) (Abcam) at $4^{\circ} \mathrm{C}$ overnight. After $1.5 \mathrm{~h}$ of incubation in horseradish peroxidase-labelled secondary antibodies (ZB-2308, ZSGB-BIO), the blots were detected using ECL western blot kits (Pierce, Rockford, IL, USA) according to the manufacturer's instructions. The density of the bands was quantified with an ImageJ Software (National Institute of Health, USA). The expression of $\beta$-actin was used as an internal control.

Histopathological and immunohistochemical analyses. Immunohistochemisty was used to detect the expression of Mortalin in 10 normal liver samples and 100 HCC tumor and corresponding paracarcinomatous samples; Vimentin and CD34 were only examined in HCC tumor tissues. Briefly, formalin-fixed and paraffin-embedded tissues were cut into sections with a thickness of $2 \mu \mathrm{m}$. Sections were stained with hematoxylin and eosin (HE) for histological examination. Sections were dewaxed in xylene and rehydrated in a graded series of alcohols. Thereafter, antigen retrieval was performed by microwaving in sodium citrate buffer $(10 \mathrm{mM}$, $\mathrm{pH}$ 6.0) for $15 \mathrm{~min}$ and endogenous peroxidase activity was blocked with 3\% hydrogen peroxide for $10 \mathrm{~min}$. Subsequently, rabbit anti-Mortalin antibody (ab53098) (Abcam), mouse antiVimentin antibody (ZM-0260, ZSGB-BIO), mouse anti-CD34 (ZM-0046,ZSGB-BIO) antibody were, respectively, incubated at $4^{\circ} \mathrm{C}$ overnight. Following $30 \mathrm{~min}$ of incubation in horseradish peroxidase-conjugated secondary antibody (PV-6000, ZSGB-BIO), sections were stained in 3, 3-diaminobenzidine tetrahydrochloride (DAB) (ZLI-9017, ZSGB-BIO) solution under microscopic observation and then counterstained 
Table I. Clinicopathological factors and the expression of Mortalin in 100 patients with HCC.

\begin{tabular}{lllll}
\hline & \multicolumn{1}{c}{$\begin{array}{c}\text { Mortalin } \\
\text { expression }\end{array}$} & & \\
Characteristics & Case & Low High & $\chi^{2}$ & P-value
\end{tabular}

\begin{tabular}{llrlll}
\hline Age (years) & & & & & \\
$\leq 55$ & 28 & 3 & 25 & 2.421 & 0.210 \\
$>55$ & 72 & 20 & 52 & &
\end{tabular}

Gender

Male

Female

$\begin{array}{rrr}76 & 19 & 57 \\ 24 & 4 & 20\end{array}$

0.322

0.570

Tumor size $(\mathrm{cm})$

$$
\leq 5
$$

$>5$

$$
5
$$

3.352

0.067

Tumor nodules

Single
Multiple

Tumor capsula

$$
\text { Complete }
$$

None

$\begin{array}{rrr}68 & 18 & 50 \\ 32 & 5 & 27\end{array}$

$0.898 \quad 0.343$

$\mathrm{AFP}(\mathrm{ng} / \mathrm{ml})$

$\leq 400$
$>400$
$\mathrm{ICGR}_{15}(\%)$
$\leq 10$
$>10$

HBsAg

$$
\text { Positive }
$$

Negative

Liver cirrhosis

Present

Absent

Child-Pugh grade

$$
\text { A }
$$

Edmondson grade

\begin{tabular}{lrrrrr} 
I-II & 67 & 20 & 47 & 4.272 & 0.039 \\
III-IV & 33 & 3 & 30 & & \\
TNM stage & & & & & \\
I-II & 64 & 21 & 43 & 8.188 & 0.004 \\
III-IV & 36 & 2 & 34 & & \\
$\begin{array}{l}\text { Invasion and } \\
\text { metastasis }\end{array}$ & & & & & \\
Absent & 61 & 19 & 42 & 4.742 & 0.029 \\
Present & 39 & 4 & 35 & & \\
\hline
\end{tabular}

$\mathrm{ICGR}_{15}$, indocyanine green retention rate at $15 \mathrm{~min}$. lightly with hematoxylin. The positive controls were used with sections of breast cancer and the negative controls were processed with PBS instead of primary antibody.

Mortalin and Vimentin staining were assessed by a semiquantitative scoring system including the intensity of staining and the proportion of stained cells (15). At low-power (x40) microscope, staining intensity of tissue sections were scored ( 0 , no staining; 1 , weak staining appearing as light yellow; 2, moderate staining appearing as yellowish-brown; 3 , strong staining appearing as brown); at high-power (x400) microscope, $>5$ fields in one section were scrutinized and then the percentage of positive stained cells was calculated $(0$, none; $1,<10 \% ; 2,10 \%-50 \% ; 3,>50 \%)$. The final score of each section was obtained: [(score for staining intensity) $\mathrm{x}$ (score for percentage of positive cells)]. For category analysis, immunoreactivity of tumor cells was distinguished between high (total score $\geq 4$ ) and low (total score $<4$ ). CD34 is an antigen present in the vascular endothelial cells. We used antibodies against CD34 to stain vascular endothelial cells and then calculated the microvessel density (MVD) (16). The field of maximal CD34 expression was found in tumor tissues. Within the field, the area of maximal angiogenesis was selected and microvessels were counted on a x200 magnification field. The immunohistochemical results were scored by three pathologists who were blinded to clinical data.

Plasmid extraction and RNA interference. The small hairpin RNAs (shRNA) (Genechem, China) against Mortalin were designed by inserting oligos with a hairpin loop into the GV115 vector. The target sequences of Mortalin shRNA (NM_004134, CTGGAATGGCCTTAGTCAT) were synthesized by Shanghai GeneChem Co. Bacteria containing the plasmid were inoculated on solid culture medium with Amp resistance in the incubator. A bacterial colony was picked and then inoculated in liquid culture medium on a vibration shaker at $37^{\circ} \mathrm{C}$ (shaker speed $150 \mathrm{rpm}$ ) overnight. Harvest overnight bacterial culture by centrifuging 12,000 $\mathrm{g}$ for 2 min. Plasmid was extracted using the Qiagen Plasmid Mini kit (Qiagen, China) according to the manufacturer's instructions, then dissolved in a suitable volume of TE buffer. MHCC $97 \mathrm{H}$ cells at $\sim 3 \times 10^{5} / \mathrm{ml}$ were seeded in a 6 -well plate and placed in antibiotic-free DMEM containing $10 \%$ FBS. When the cells confluence reached $90 \%$, transient transfection of MHCC97H cells was performed. A mixture containing $10 \mu \mathrm{l} /$ well Lipofectamine 2000 (Invitrogen), $10 \mu \mathrm{l} /$ well plasmid and $480 \mu \mathrm{l} /$ well OPTI-MEM were added to plates. After $6 \mathrm{~h}$, OPTI-MEM was replaced by DMEM containing $10 \%$ FBS.

Three groups were established in this experiment: blank group (no interference), NC (negative control) group (transfected with NC shRNA) and shRNA group (transfected with Mortalin shRNA). Cells were harvested 24, 48, 72 and $96 \mathrm{~h}$ after transfection and subjected to MTT assay, flow cytometry, qPCR and western blot analyses. Simultaneously, a GFP (green fluorescent protein) plasmid was used to determine transfection efficiency and cell viability was determined by the MTT assay (MTT Cell Proliferation and Cytotoxicity Assay kit) and flow cytometry (Annexin V/PI apoptosis kit). All experiments were performed in triplicate to confirm the reproducibility. 
Table II. Relative quantity of Mortalin mRNA and protein in six cell lines.

\begin{tabular}{ccccccc}
\hline & Hep3B & MHCC97H & HepG2 & L02 & MHCC97L & HCCLM3 \\
\hline mRNA & $0.45 \pm 0.08$ & 1 & $0.14 \pm 0.06$ & $0.04 \pm 0.01$ & $0.05 \pm 0.02$ & $2.38 \pm 0.27$ \\
Protein & $0.72 \pm 0.11$ & $1.21 \pm 0.13$ & $0.35 \pm 0.07$ & 0 & 0 & $2.14 \pm 0.29$ \\
\hline
\end{tabular}

MTT assay [3-(4,5-dimethylthiazol-2-yl)-2,5-diphenyltetrazolium bromide]. Twenty-four hours post-transfection cells $\left(2.0 \times 10^{4} / \mathrm{ml}, 200 \mu \mathrm{l} /\right.$ well) were cultured in 96 -well plates with DMEM among three groups. MTT $(0.5 \%)$ (Beyotime, China) with a volume of $20 \mu \mathrm{l}$ was added to each well for $4 \mathrm{~h}$ and then DMEM was aspirated from wells as far as possible without disturbing the cells and crystals on the plastic surface. Subsequently, each well was added with a $200-\mu 1$ dimethyl sulfoxide (DMSO) to dissolve crystals and the plate agitated on a plate shaker for $10 \mathrm{~min}$. The optical density (OD) measurements were carried out in an enzyme-linked immunosorbent assay (ELISA) reader at the wavelength of $490 \mathrm{~nm}$. The reader was calibrated to 0 absorbance using DMEM without cells.

Flow cytometry. Twenty-four hours post-transfection, cells $\left(2.0 \times 10^{5}\right)$ were collected by centrifugation among three groups and then suspended in $500 \mu 11 \mathrm{X}$ binding buffer. Subsequently, $5 \mu \mathrm{l}$ Annexin V-FITC and $10 \mu \mathrm{l}$ propidium iodide (PI) (Annexin V/PI apoptosis kit, Multi Sciences) were added to each sample. Samples were gently mixed and incubated at room temperature for $5 \mathrm{~min}$ in the dark. The cells were analyzed on a Canto-II ${ }^{\mathrm{TM}}$ reader (BD Biosciences) and data were analyzed with FlowJo software (Tree Star, Ashland, OR, USA).

Statistical analysis. Statistical analyses were performed using the statistical package SPSS 13.0 (SPSS Inc., Chicago, IL, USA). The difference in Mortalin mRNA and protein expression level among six cell lines and tissue samples were examined using Student's t-test. Fisher's exact or $\chi^{2}$ tests were performed to analyze correlations among different protein expressions or between protein expressions and various clinicopathological parameters. $\mathrm{P}<0.05$ was considered statistically significant.

\section{Results}

The expression level of Mortalin $\mathrm{mRNA}$ and protein in HCC and normal liver cell lines. The expression of nodal mRNA was detected in all cell lines. The expression of Mortalin protein was detected in Hep3B, MHCC97H, HepG2 and HCCLM3, but not in MHCC97L and L02 (Fig. 1). The mean expression level of Mortalin mRNA and protein are shown in Table II. It was found that among the three human HCC cell lines with the same genetic background, the expression level of Mortalin was the highest in HCCLM3, while it was the lowest in MHCC97L $(\mathrm{P}<0.05)$.

The expression level of Mortalin $\mathrm{mRNA}$ and protein in HCC tumor, paracarcinomatous and normal liver tissues. Mortalin was detected in 10 normal liver samples and all $13 \mathrm{HCC}$
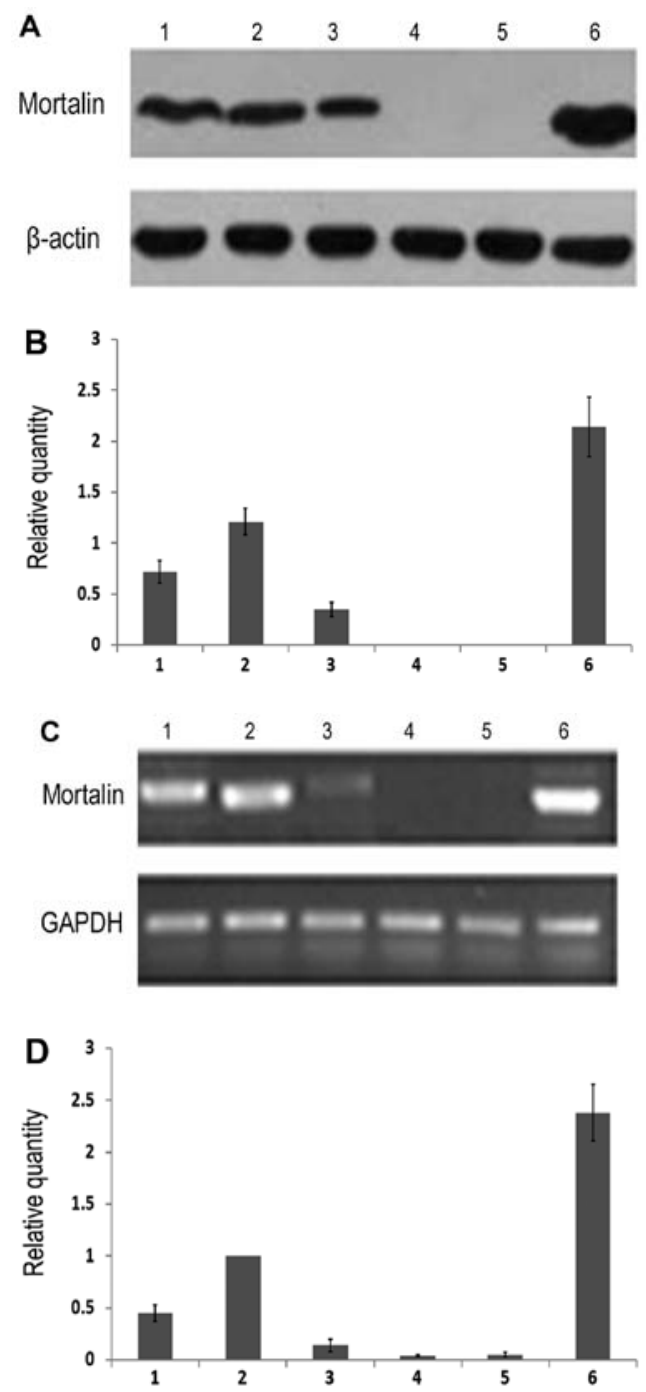

Figure 1. Expression of Mortalin protein and mRNA in liver cell lines. 1, Hep3B; 2, MHCC97H; 3, HepG2; 4, L02; 5, MHCC97L; 6, HCCLM3. (A) The expression of Mortalin protein in six cell lines using western blotting. (B) Relative quantity of Mortalin protein expressed in six cell lines. (C) The expression of Mortalin mRNA in six cell lines using qPCR. (D) Relative quantity of Mortalin mRNA expressed in six cell lines.

tumors and corresponding paracarcinomatous samples. The mean mRNA and protein level of Mortalin in HCC tumor, paracarcinomatous and normal liver tissues were $1,0.36 \pm 0.14$, $0.18 \pm 0.07$ and $2.58 \pm 0.21,0.22 \pm 0.09,0.05 \pm 0.02$, respectively (Fig. 2). The results showed that the expression level of Mortalin in HCC tumor tissues was significantly higher than that in paracarcinomatous and normal tissues $(\mathrm{P}<0.05)$.

Immunohistochemical expression of Mortalin in HCC tumor, paracarcinomatous and normal liver tissues. We found that 

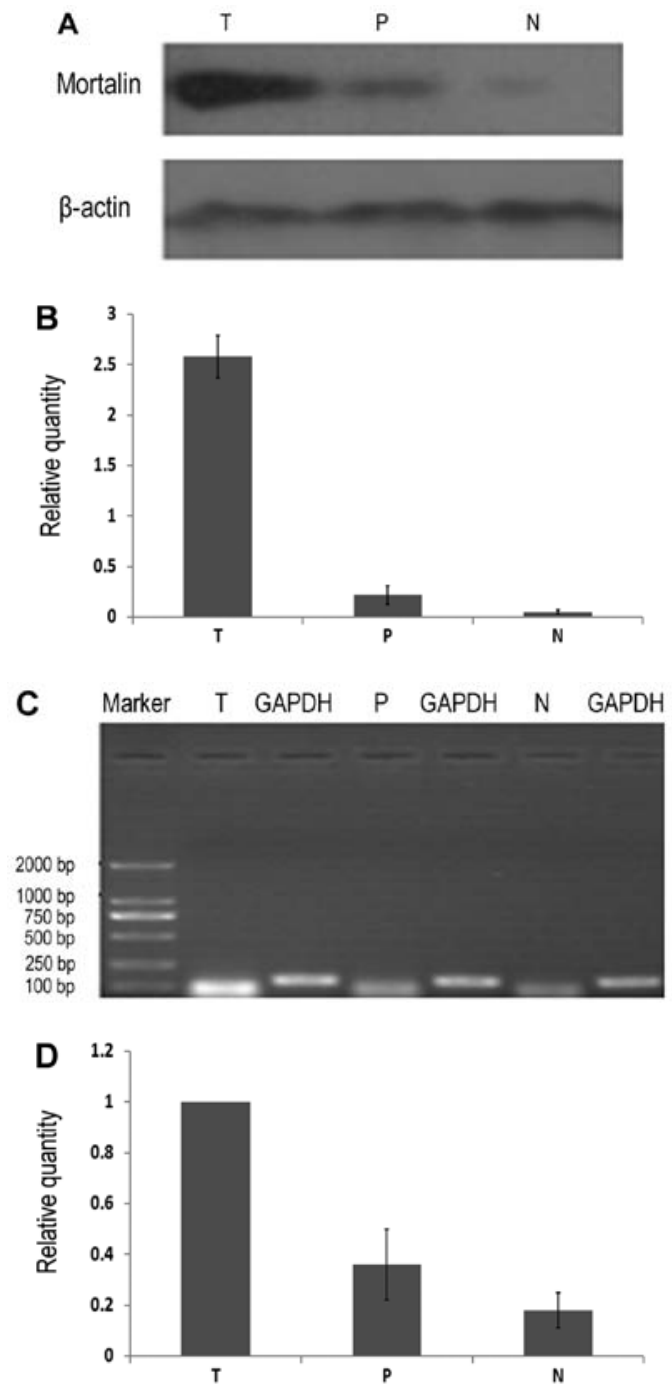

Figure 2. Expression of Mortalin protein and mRNA in liver tissues. T, HCC tumor tissue; $\mathrm{P}$, paracarcinomatous tissue; N, normal tissue. (A) Expression level of Mortalin protein in liver tissues using western blotting. (B) Relative quantity of Mortalin protein expressed in liver tissues. (C) Expression level of Mortalin mRNA in liver tissues using qPCR. (D) Relative quantity of Mortalin mRNA expressed in liver tissues.

positive expression of Mortalin was mainly cytoplasmic, some tumor cells stained strongly, while others exhibited slight or no staining at all (Fig. 3B-D). Mortalin expression was detected in $77 \%$ (77/100) of HCC tumor tissues, which was significantly higher than $19 \%(19 / 100)$ in the paracarcinomatous tissues and $1 \%(1 / 10)$ in the normal liver tissues $\left(\chi^{2}=67.388, \mathrm{P}<0.001\right.$; $\chi^{2}=16.669, \mathrm{P}<0.001$, respectively) (Table III). Association between the expression of Mortalin and clinicopathological characteristics was evaluated and was found that the high expression of Mortalin was positively correlated with poor Edmondson grade $\left(\chi^{2}=4.272, \mathrm{P}=0.039\right)$, advanced TNM stages $\left(\chi^{2}=8.188, \mathrm{P}=0.004\right)$ and status of invasion and metastasis $\left(\chi^{2}=4.742, \mathrm{P}=0.029\right)$, but there was no significant correlation with other variables (Table I).

Immunohistochemical expression of Mortalin, Vimentin and MVD in HCC tumor tissues. Vimentin is an important hallmark of EMT; high expression of Vimentin was observed
Table III. Immunohistochemical expression of Mortalin in $\mathrm{HCC}$, paracarcinomatous tissues and normal liver tissues.

\begin{tabular}{lrrrr}
\hline & \multicolumn{4}{c}{ Mortalin expression } \\
\cline { 2 - 4 } Liver tissue & Low & High & $\chi^{2}$ & P-value \\
\hline HCC tumor tissue & 23 & 77 & & \\
Paracarcinomatous tissue & 81 & 19 & 67.388 & $<0.001$ \\
Normal tissue & 9 & 1 & 16.669 & $<0.001$
\end{tabular}

Table IV. Correlation between Mortalin and Vimentin expression in $100 \mathrm{HCC}$ patients with HCC.

\begin{tabular}{lccccc}
\hline & \multicolumn{3}{l}{ Vimentin expression } & & \\
\cline { 2 - 3 } Mortalin expression & Low & High & r & P-value \\
\hline Low & 16 & 7 & 0.236 & 0.018 \\
High & 32 & 45 & & \\
\hline
\end{tabular}

mainly in the cytoplasmic staining (Fig. 3G and H). Among all patients with HCC, 52\% (52/100) patients had high expression of Vimentin. Spearman's rank correlation test was used to analyze the relation between Mortalin and Vimentin; a significant positive correlation was found between the expression of Mortalin and Vimentin $(r=0.236, \mathrm{P}=0.018)$ (Table IV). Angiogenesis is an important process of growth and metastasis in HCC; MVD was detected by using an antibody against CD34 (Fig. 3E and F). MVD in HCC tumor tissues ranged from 0 to 139/200 x field (median, 32/200 x field). We found that tumors with high expression of Mortalin had a tendency to higher MVD than those with the low expression of Mortalin (39.4 \pm 42.5 vs. $29.7 \pm 16.9, \mathrm{t}=1.630, \mathrm{P}=0.106)$.

shRNA transfection in MHCC97H cells. GFP fluorescence showed that plasmid Mortalin shRNA was successfully transfected into MHCC97H cells (Fig. 4). MTT and flow cytometry assays were applied to determine cell viability $24 \mathrm{~h}$ after transfection among the three groups. OD values were not significantly different among the three groups (P>0.05) (Fig. 5). The result showed that only 2.5 and $3.5 \%$ cytotoxicity was present compared to the blank group. Similarly, flow cytometry analysis showed that early apoptosis (Annexin $\mathrm{V}^{+} / \mathrm{PI}^{-}$) rates among the three groups were $0.8,4.5$ and $9.2 \%$ (Fig. 6), indicating that transfection did not cause severe cell damage. To demonstrate the level of inhibition after transfection, qPCR assay was performed. As shown in Fig. 7A, Mortalin mRNA level of cells were $2.52 \pm 0.37,1.97 \pm 0.28,1.12 \pm 0.25$ and $0.55 \pm 0.13$ in shRNA group after 24, 48, 72 and 96 h, respectively. Simultaneously, the expression level of Mortalin mRNA in blank group and NC group were $2.90 \pm 0.31,2.83 \pm 0.22,2.95 \pm 0.27,2.90 \pm 0.23$ and $2.88 \pm 0.41,2.80 \pm 0.15,2.93 \pm 0.30,2.91 \pm 0.09$, respectively. The expression level of Mortalin mRNA in shRNA group was significantly lower than that in blank group and NC group $(\mathrm{P}<0.05$, respectively). These results showed that Mortalin 

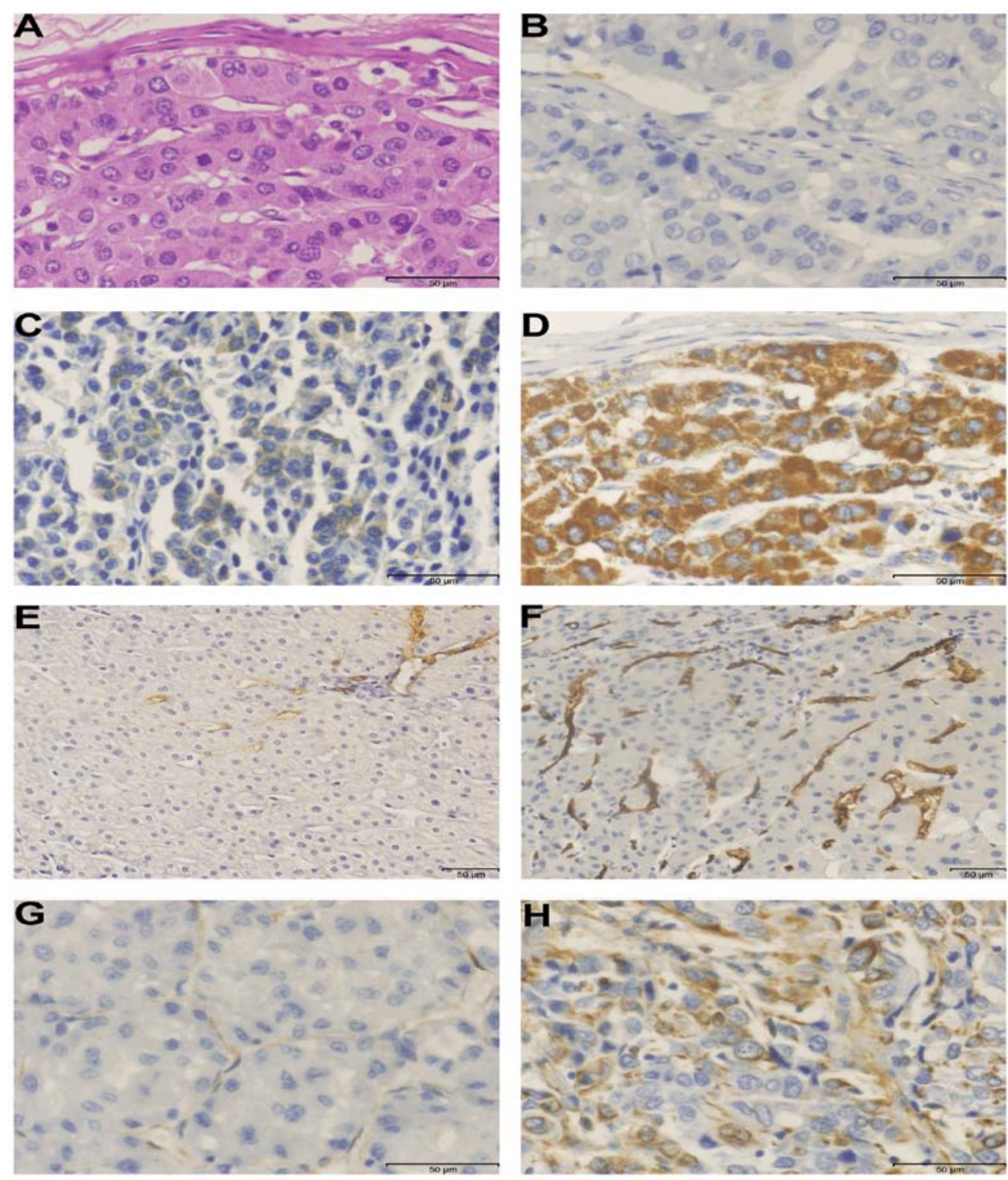

Figure 3. Immunohistochemical staining of Mortalin, CD34 and Vimentin in HCC tumors. (A) HCC tumor, hematoxylin-eosin staining (x400). (B) Negative expression of Mortalin (x400). (C) Low expression of Mortalin (x400). (D) High expression of Mortalin (x400). (E) Low expression of MVD (x200). (F) High expression of MVD (x200). (G) Low expression of Vimentin (x400). (H) High expression of Vimentin (x400).
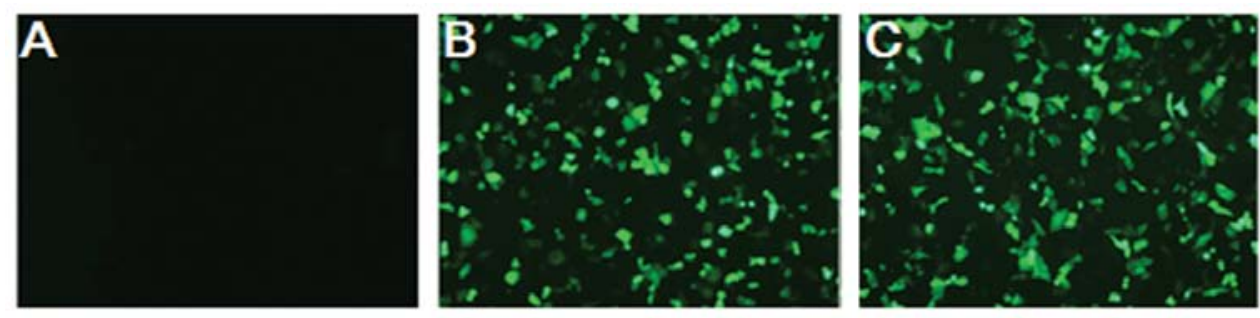

Figure 4. Green fluorescence in MHCC97H cells $24 \mathrm{~h}$ after transfection (x200) in the blank group (A), NC group (B) and siRNA group (C).

shRNA was an effective sequence to suppress the target gene and the capability of interference increased gradually upto $96 \mathrm{~h}$ after transfection.

To find the relationship between Mortalin and Vimentin, qPCR and western blot assays were applied. Vimentin mRNA level of cells in shRNA group after 24, 48, 72 and $96 \mathrm{~h}$ were
$1.56 \pm 0.21,1.02 \pm 0.27,0.58 \pm 0.16$ and $0.31 \pm 0.07$, respectively (Fig. 7A). 24, 48, 72 and $96 \mathrm{~h}$ after transfection, the expression level of Mortalin protein and Vimentin protein in shRNA group were $2.09 \pm 0.37,1.48 \pm 0.23,0.73 \pm 0.11,0.25 \pm 0.06$ and $1.03 \pm 0.21,0.54 \pm 0.14,0.21 \pm 0.07,0.11 \pm 0.02$, respectively (Fig. 7B). We found that the decreased expression of Mortalin 


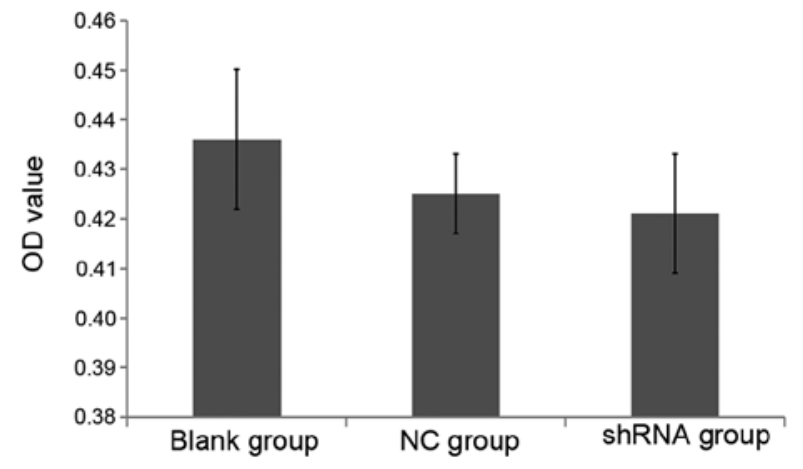

Figure 5 . Cell viability (OD value) was measured by MTT assays $24 \mathrm{~h}$ after transfection in the blank group, NC group and siRNA group, respectively.
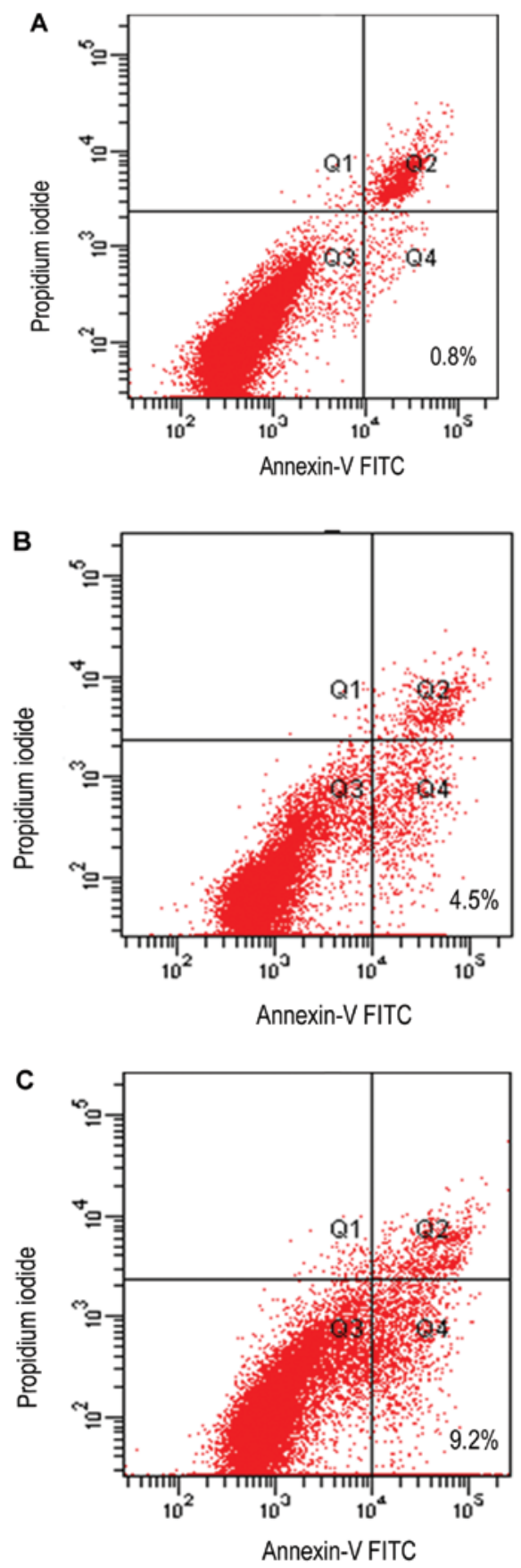

Figure 6. Cells in the blank group (A), NC group (B) and siRNA group (C) were analyzed by flow cytometry $24 \mathrm{~h}$ after transfection.
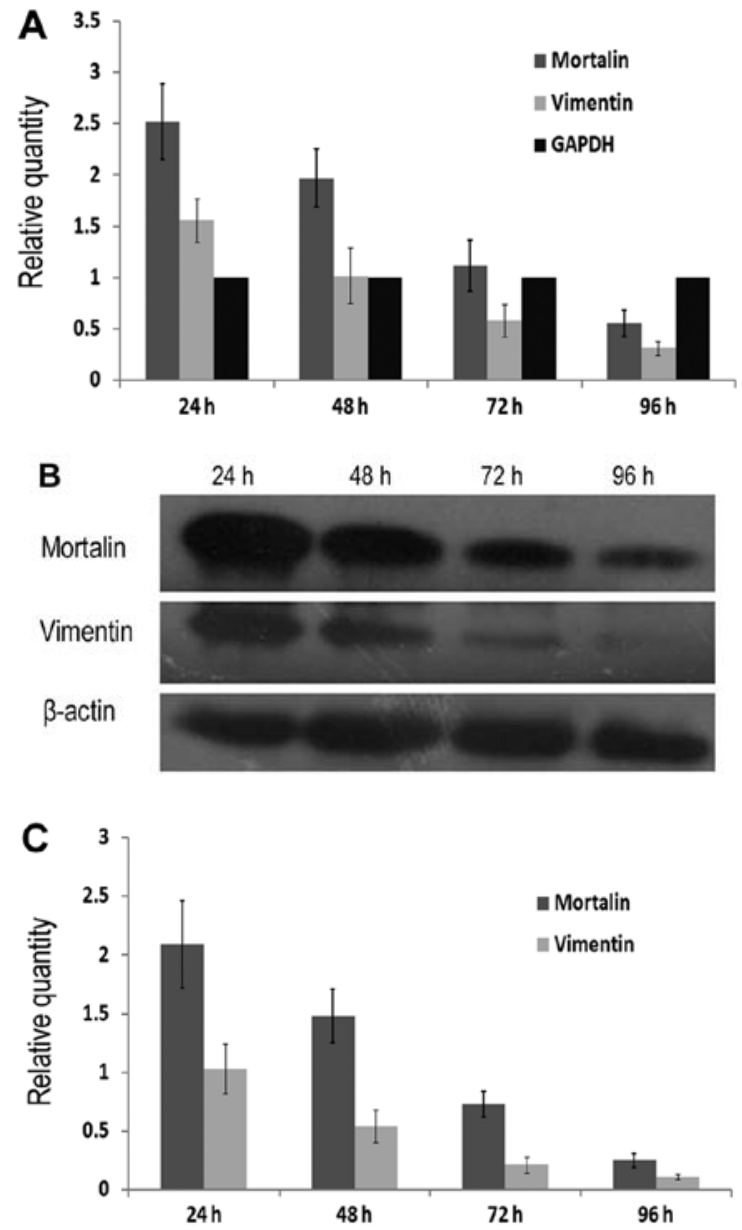

Figure 7. The expression level of Mortalin and Vimentin in MHCC97H cells. 1, shRNA group for $24 \mathrm{~h} ; 2$, shRNA group for $48 \mathrm{~h} ; 3$, shRNA group for $72 \mathrm{~h} ; 4$, shRNA group for $96 \mathrm{~h}$. (A) Relative quantity of Mortalin mRNA and Vimentin mRNA expressed in MHCC97H cells. (B) The expression of Mortalin protein and Vimentin protein were measured by western blotting. (C) Relative quantity of Mortalin protein and Vimentin protein expressed in MHCC $97 \mathrm{H}$ cells.

was accompanied by a reduction of Vimentin expression (Fig. 7C). This observation supported our conclusion that inhibition of Mortalin expression could decrease the expression of Vimentin and had a suppressive function in epithelial-tomesenchymal transition.

\section{Discussion}

HCC invasion and metastasis are closely related to adverse clinical outcome and shorter survival of cancer patients. Although great efforts have been made to clarify molecular mechanisms involved in HCC invasion and metastasis, the detailed mechanisms for HCC malignancy and metastasis are still unknown. It was reported that angiogenesis and EMT played important roles in the occurrence and progression of HCC $(16,17)$. Mortalin, as a 'stress protein', was a highly conserved molecular chaperone and abundant in the same tumors and played a key role in cell cycle regulation, carcinogenesis and tumor progression $(7,18,19)$. Simultaneously, Mortalin-p53 interaction resulted in inhibition of transcriptional activation and control of centrosome 
duplication functions $(9,20)$. In addition, Lu et al $(21)$ found that inhibitor of Mortalin was able to induce tumor cell apoptosis. Therefore, Mortalin was suggested to serve as a therapeutic marker.

It was previously reported that Mortalin was overexpressed in leukemia, brain tumors, colon carcinoma and tumor cell lines $(7,22)$. Yi et al (11) have demonstrated that overexpression of Mortalin was closely associated with venous infiltration and advanced tumor stages in HCC. These findings suggested that overexpressed Mortalin could increase malignancy and aggressive behavior and predicted early recurrence in HCC. Chen et al (23) found that expression of Mortalin was notably higher in the SMMC 7721 than in a normal liver cell line. Lu et al (21) showed that human HepG2 cells lacked mortalin-p53 interaction and were resistant to apoptosis, but cell apoptosis was significantly increased by Mortalin shRNA transfection. These findings showed the potential for clinical application of chemotherapeutic drugs in HCC treatment.

HCC is usually a hypervascular tumor and upregulation of Vimentin is considered as the essential step of EMT process. When a liver tumor was $1-2 \mathrm{~mm}$ in diameter, it could obtain nutrients through diffusion without an extensive vasculature. If tumor growth become much larger, angiogenesis must occur, also, metastasis is required for angiogenesis (24). CD34 is considered to be a marker for neovascularization, the expression of CD34 positive endothelial cells played an important role in the process of angiogenesis in HCC and metastasis. EMT was first discovered at key transition steps during embryogenesis and was the critical event that mediated tumor metastasis. Through activation of several specific transcription factors, tumor cells invariably adopt a mesenchymal phenotype to invade surrounding tissues and metastasize $(4,25)$. Vimentin has been recognized as a marker for EMT and its overexpression has been strongly associated with metastatic phenotype and poor prognosis.

To our knowledge, this was the first study to investigate the relationship among Mortalin expression, angiogenesis and EMT by immunohistochemical staining. We discovered that Mortalin had a significant correlation with Vimentin, but the high expression of Mortalin group did not have significantly higher MVD than that in low expression of Mortalin group. This shows that Mortalin could not promote angiogenesis, but could contribute to the process of EMT. We also observed that the expression level of Mortalin in HCCLM3 with the highest metastatic potential was notably higher than in the other five cell lines with lower metastatic potential. This suggested that Mortalin expression correlated with the metastatic phenotype of HCC cells.

In three cell lines with the same genetic background and various metastatic potentials, HCCLM3, with the highest metastatic potential, exhibited the highest level of Mortalin and MHCC97L, with the lowest metastatic potential, having the lowest level of Mortalin. This showed that overexpression of Mortalin could possess metastasis-inducing capabilities. We analyzed expression of Mortalin and clinicopathological characteristics and found Mortalin was associated with Edmondson grade, TNM stage and tumor invasion and metastasis. By Mortalin shRNA transfection, we also found that decreased expression of Mortalin was accompanied by a reduction of Vimentin expression. Our conclusions are that low expression of Mortalin was able to inhibit EMT and decrease tumor progression and lose the metastasis-inducing capability.

Although the same studies have identified that HSP70 blocked TGF- $\beta$-induced EMT in HaCat cells (26) and HSP72 inhibited EMT by inhibiting Smad3 activation in renal epithelial cells (27), the mechanisms by which Mortalin (HSP75) promoted EMT and tumor metastasis are still unclear. To solve these problems, further experiments are required.

In conclusion, our study suggests that overexpression of Mortalin is correlated with metastatic phenotype of HCC cells and can promote EMT, but cannot induce angiogenesis in HCC. The decreased expression of Mortalin is accompanied by an inhibition of EMT in the HCC cell line. Using shRNA transfection, Mortalin knockdown may have potential for clinical application to decrease tumor metastasis and recurrence after curative surgery.

\section{Acknowledgements}

This study was supported by the National Natural Science Foundation of China (No. 81272398), the Science and Technological Fund of Anhui Province (No. 12010402112) and the Anhui Provincial Natural Science Foundation of Institution of Higher Education (No. KJ2011A171).

\section{References}

1. Jemal A, Bray F, Center MM, Ferlay J, Ward E and Forman D: Global cancer statistics. CA Cancer J Clin 61: 69-90, 2011.

2. Forner A, Llovet JM and Bruix J: Hepatocellular carcinoma. Lancet 379: 1245-1255, 2012.

3. Polyak K and Weinberg RA: Transitions between epithelial and mesenchymal states: acquisition of malignant and stem cell traits. Nat Rev Cancer 9: 265-273, 2009.

4. Christiansen JJ and Rajasekaran AK: Reassessing epithelial to mesenchymal transition as a prerequisite for carcinoma invasion and metastasis. Cancer Res 66: 8319-8326, 2006.

5. Tanaka S and Arii S: Molecular targeted therapies in hepatocellular carcinoma. Semin Oncol 39: 486-492, 2012.

6. Wadhwa R, Taira K and Kaul SC: An Hsp70 family chaperone, mortalin/mthsp70/PBP74/Grp75: what, when, and where? Cell Stress Chaperones 7: 309-316, 2002.

7. Wadhwa R, Takano S, Kaur K, et al: Upregulation of mortalin/ mthsp70/Grp75 contributes to human carcinogenesis. Int J Cancer 118: 2973-2980, 2006.

8. Mizukoshi E, Suzuki M, Misono T, et al: Cell-cycle dependent tyrosine phosphorylation on mortalin regulates its interaction with fibroblast growth factor-1. Biochem Biophys Res Commun 280: 1203-1209, 2001.

9. Wadhwa R, Takano S, Robert M, et al: Inactivation of tumor suppressor p53 by mot-2, a hsp70 family member. J Biol Chem 273: 29586-29591, 1998.

10. Wadhwa R, Yaguchi T, Hasan MK, Taira K and Kaul SC: Mortalin-MPD (mevalonate pyrophosphate decarboxylase) interactions and their role in control of cellular proliferation. Biochem Biophys Res Commun 302: 735-742, 2003.

11. Yi X, Luk JM, Lee NP, et al: Association of mortalin (HSPA9) with liver cancer metastasis and prediction for early tumor recurrence. Mol Cell Proteomics 7: 315-325, 2008.

12. Li Y, Tang Y, Ye L, et al: Establishment of a hepatocellular carcinoma cell line with unique metastatic characteristics through in vivo selection and screening for metastasis-related genes through cDNA microarray. J Cancer Res Clin Oncol 129: 43-51, 2003.

13. Li Y, Tang ZY, Ye SL, et al: Establishment of cell clones with different metastatic potential from the metastatic hepatocellular carcinoma cell line MHCC97. World J Gastroenterol 7: 630-636, 2001. 
14. Edmondson HA and Steiner PE: Primary carcinoma of the liver: a study of 100 cases among 48,900 necropsies. Cancer 7: 462-503, 1954.

15. Chen MH, Yip GW, Tse GM, et al: Expression of basal keratins and vimentin in breast cancers of young women correlates with adverse pathologic parameters. Mod Pathol 21: 1183-1191, 2008.

16. Weidner N, Semple JP, Welch WR and Folkman J: Tumor angiogenesis and metastasis - correlation in invasive breast carcinoma. N Engl J Med 324: 1-8, 1991.

17. Thiery JP: Epithelial-mesenchymal transitions in tumour progression. Nat Rev Cancer 2: 442-454, 2002.

18. Hartl FU: Molecular chaperones in cellular protein folding. Nature 381: 571-579, 1996.

19. Calderwood SK, Khaleque MA, Sawyer DB and Ciocca DR Heat shock proteins in cancer: chaperones of tumorigenesis. Trends Biochem Sci 31: 164-172, 2006.

20. Ma Z, Izumi H, Kanai M, Kabuyama Y, Ahn NG and Fukasawa K: Mortalin controls centrosome duplication via modulating centrosomal localization of p53. Oncogene 25: 5377-5390, 2006.
21. Lu WJ, Lee NP, Kaul SC, et al: Mortalin-p53 interaction in cancer cells is stress dependent and constitutes a selective target for cancer therapy. Cell Death Differ 18: 1046-1056, 2011.

22. Dundas SR, Lawrie LC, Rooney PH and Murray GI: Mortalin is over-expressed by colorectal adenocarcinomas and correlates with poor survival. J Pathol 205: 74-81, 2005.

23. Chen $\mathrm{X}, \mathrm{Xu} \mathrm{B}, \mathrm{Li} \mathrm{H}$, et al: Expression of mortalin detected in human liver cancer by tissue microarrays. Anat Rec 294: 1344-1351, 2011.

24. Kimura H, Nakajima T, Kagawa K, et al: Angiogenesis in hepatocellular carcinoma as evaluated by CD34 immunohistochemistry. Liver 18: 14-19, 1998.

25. Gupta GP and Massague J: Cancer metastasis: building a framework. Cell 127: 679-695, 2006.

26. Zhou Y, Mao H, Li S, et al: HSP72 inhibits Smad3 activation and nuclear translocation in renal epithelial-to-mesenchymal transition. J Am Soc Nephrol 21: 598-609, 2010.

27. Li Y, Kang X and Wang Q: HSP70 decreases receptor-dependent phosphorylation of Smad2 and blocks TGF-beta-induced epithelial-mesenchymal transition. J Genet Genomics 38: 111-116, 2011. 